\title{
The Impact of Latency on Perceptual Judgments and Motor Performance in Closed-loop Interaction in Virtual Reality
}

\author{
Thomas Waltemate $^{1} \quad$ Irene Senna $^{2} \quad$ Felix Hülsmann ${ }^{1,3}$ Marieke Rohde ${ }^{4}$ \\ Stefan Kopp ${ }^{3}$ Marc Ernst $^{2} \quad$ Mario Botsch ${ }^{1}$ \\ ${ }^{1}$ Computer Graphics Group, Bielefeld University, Germany $\quad{ }^{2}$ Applied Cognitive Psychology, Ulm University, Germany \\ ${ }^{3}$ Social Cognitive Systems, Bielefeld University, Germany $\quad{ }^{4}$ AFFS Affective Signals GmbH, Berlin, Germany
}
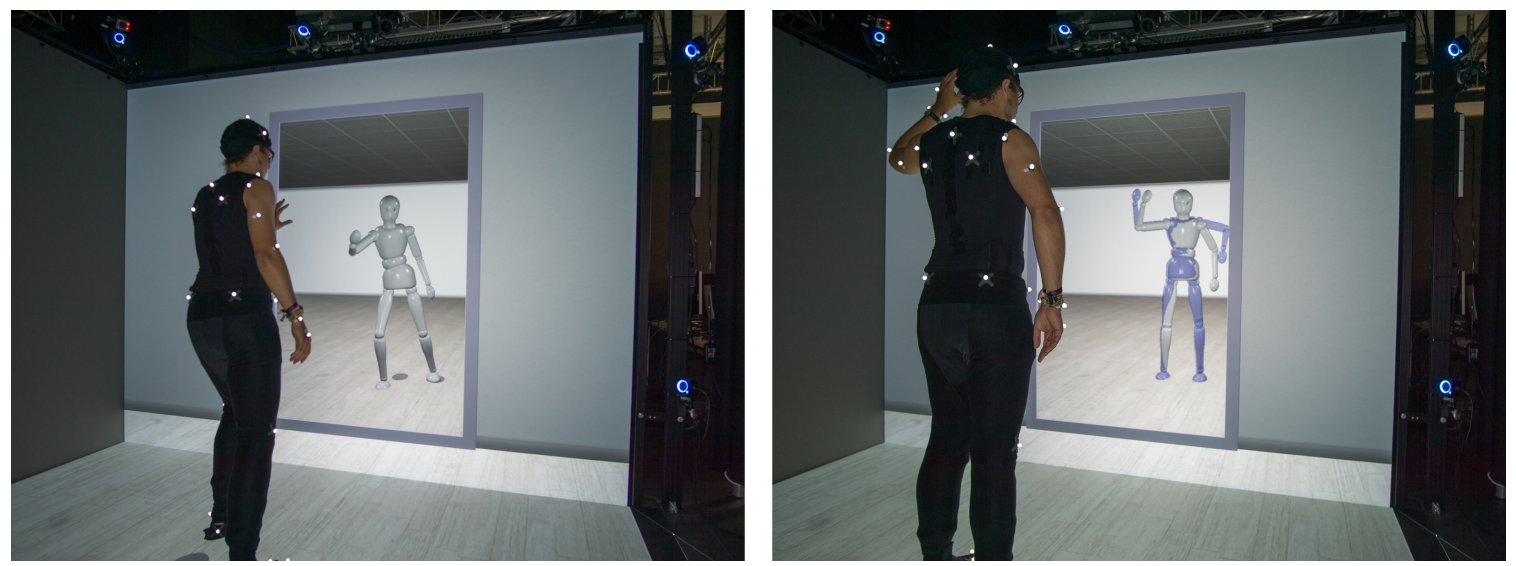

Figure 1: Left: The basic virtual mirror scenario consists of an empty room and a simplistic mirror avatar. Right: The extended scenario employed in the experiment, where the target movement is shown by a semi-transparent blue "ghost character".

\begin{abstract}
Latency between a user's movement and visual feedback is inevitable in every Virtual Reality application, as signal transmission and processing take time. Unfortunately, a high end-to-end latency impairs perception and motor performance. While it is possible to reduce feedback delay to tens of milliseconds, these delays will never completely vanish. Currently, there is a gap in literature regarding the impact of feedback delays on perception and motor performance as well as on their interplay in virtual environments employing full-body avatars. With the present study at hand, we address this gap by performing a systematic investigation of different levels of delay across a variety of perceptual and motor tasks during full-body action inside a Cave Automatic Virtual Environment. We presented participants with their virtual mirror image, which responded to their actions with feedback delays ranging from 45 to $350 \mathrm{~ms}$. We measured the impact of these delays on motor performance, sense of agency, sense of body ownership and simultaneity perception by means of psychophysical procedures. Furthermore, we looked at interaction effects between these aspects to identify possible dependencies. The results show that motor performance and simultaneity perception are affected by latencies above $75 \mathrm{~ms}$. Although sense of agency and body ownership only decline at a latency higher than $125 \mathrm{~ms}$, and deteriorate for a latency greater than $300 \mathrm{~ms}$, they do not break down completely even at the highest tested delay. Interestingly, participants perceptually infer the presence of delays more from their motor error in the task than from the actual level of delay. Whether or not participants notice a delay in a virtual environment might therefore depend on the motor task and their performance rather than on the actual delay.
\end{abstract}

Keywords: latency, simultaneity perception, body ownership, sense of agency, full-body motion capture, virtual mirror

Concepts: •Computing methodologies $\rightarrow$ Virtual reality;

\section{Introduction}

Virtual Reality (VR) plays an essential role in various fields, such as training of emergency situations, simulations, rehabilitation, or motor learning. The success of VR applications is determined by many factors, such as realism, ease of interaction, and responsiveness [Meehan et al. 2003]. Among these, one crucial factor is the end-to-end latency: the delay between a user's interaction and the corresponding feedback presentation. This holds especially for applications in which users obtain real-time feedback of their action, as is the case when they are presented with their own avatar. In setups presenting delayed visual feedback with respect to motor performance, high latencies influence perceived temporal coherence of the scene, sense of agency, sense of ownership, as well as motor performance [Franck et al. 2001; Longo and Haggard 2009; Jörg et al. 2012; Imaizumi and Asai 2015]. If we had knowledge on

\footnotetext{
Permission to make digital or hard copies of all or part of this work for personal or classroom use is granted without fee provided that copies are not made or distributed for profit or commercial advantage and that copies bear this notice and the full citation on the first page. Copyrights for components of this work owned by others than the author(s) must be honored. Abstracting with credit is permitted. To copy otherwise, or republish, to post on servers or to redistribute to lists, requires prior specific permission and/or a fee. Request permissions from permissions@acm.org. (C) 2016 Copyright held by the owner/author(s). Publication rights licensed to ACM.

VRST '16, November 02 - 04, 2016, Garching bei München, Germany ISBN: 978-1-4503-4491-3/16/11
}

DOI: http://dx.doi.org/10.1145/2993369.2993381 
which duration of latencies is still acceptable with respect to these factors, we would be able to design systems that reduce the risk of suffering from latency.

Even though numerous studies investigating the effects of feedback delays in virtual environments have previously been conducted, no clear picture has emerged. While Held and Durlach [1991] report that delays as small as $60 \mathrm{~ms}$ significantly interfere with motor adaptation, others reported a threshold of $120 \mathrm{~ms}$ or $150 \mathrm{~ms}$ to perceive latency (e.g., [Mauve et al. 2004; Jörg et al. 2012]). Furthermore, the impact of latency is often only considered with respect to one single factor (e.g., perception of simultaneity [Mania et al. 2004; Raaen et al. 2014]). In other cases, only very simple tasks such as button pressing are examined without a focus on VR [Kawabe et al. 2013; Rohde et al. 2014a]. Also, Rohde et al. [2014b] found that feedback delays are processed differently in predictable motor tasks as opposed to unpredictable motor tasks. The effect of feedback delays is thus contingent on the display, the movement performed, and on the perceptual or motor task (see Section 2 for a review of the background literature). Most studies to date have focused on just one task and one movement, which makes it difficult to compare results from different studies and to derive acceptable levels of delay in VR across applications.

We revisit the topic of visual feedback delays in VR using a more encompassing approach. To this end, we use a virtual mirror scenario with full-body motion capture, as is employed in many virtual reality experiments where monitoring the own movement of the full body is important [Gonzalez-Franco et al. 2010; Lugrin et al. 2015; de Kok et al. 2015; Samaraweera et al. 2015]. As our virtual environment we use a Cave Autonomous Virtual Environment (CAVE), which is our preferred environment in the context of motor learning in VR [Waltemate et al. 2015]. We use this system in the project ICSPACE $^{1}$, which develops a virtual coaching system for motor learning. For such a system it is important to be able to monitor one's own body and to minimize the amount of disturbing hardware attached to the user while still providing an immersive experience. In this context, a CAVE offers a good trade-off between the amount of attached hardware and immersion. The virtual mirror image allows to continuously monitor the own full-body movements.

Our study contributes to a better understanding of the effects of feedback latencies on full-body closed-loop behavior. It combines three important factors:

Plausibility: Our VR system provides an immersive, full-body virtual mirror image and allows for full-body motor learning of complex tasks. Participants perform a motor task that requires fast and precise movements of the whole upper body. Thus, our study contributes to the development of VR environments dedicated to motor learning, such as sports training, and will hopefully be useful for the design of VR environments.

Temporal precision: Our CAVE is characterized by a low baseline end-to-end latency of $45 \mathrm{~ms}$, which is particularly low for an environment using full-body motion capturing. This allows us to evaluate our dependent variables using latencies of $45 \mathrm{~ms}$ and higher. In the present study, we investigate a range of delays, from 45 to $350 \mathrm{~ms}$, which allows us to model the perceptual responses as a continuous psychometric function of the feedback delay. Testing latencies as small as 45$125 \mathrm{~ms}$ allows us to study the impact of even small feedback delays in VR systems on perception and behavior. Exploring high latencies allows us to identify the limits of the temporal windows of perceived simultaneity, agency, and ownership in VR while performing complex movements. This gives us the

\footnotetext{
${ }^{1}$ http://graphics.uni-bielefeld.de/research/icspace/
}

opportunity to compare our results on feedback delays in immersive VR with previous related work on visual feedback delays in simpler tasks and environments, such as button presses and manual control interfaces (e.g., [Farrer et al. 2013; Rohde et al. 2014a].

Range of measures: Apart from motor performance error, we record participants' perceptual judgments (simultaneity, agency, and body ownership) after performing movements in our experimental setup. We test for interaction effects between these variables and pre-existing immersive tendencies.

This paradigm enables us to identify thresholds for tolerable delays in different domains of user performance and experience, to compare these thresholds across measures and to identify interactions between measures. We hope that our study will be a useful resource for the design of virtual environments and provides information about how transmission delays impair different aspects of human perception and performance.

\section{Related Work}

In the physical world, causes and effects of an action are temporally contiguous. The human brain automatically compensates for small and natural delays introduced by inconsistencies in neural propagation across signals in different modalities, and between movement outputs and afferent signals. In VR setups and in computer games, the latency of the system always introduces further delay between motor commands and visual feedback [Stauffert et al. 2016]. While the brain tolerates small but perceivable delays, and even temporally recalibrates to them [Rohde et al. 2014b; Yarrow et al. 2013], larger delays might affect perception and behavior. There is no golden rule specifying the highest acceptable latency a system is allowed to have in order not to impair motor and perceptual performances. Studies involving different setups and sensorimotor tasks come to different conclusions when investigating the perceptual threshold for delay detection. Users might be unaware of delays below $120 \mathrm{~ms}$ [Mauve et al. 2004], or below $150 \mathrm{~ms}$ when controlling characters in computer games [Jörg et al. 2012]. Even delays around $200 \mathrm{~ms}$ between mouse movements and cursor movements or between a button press and a visual stimulus might go unnoticed [Raaen et al. 2014; Rohde et al. 2014a]. Although the available body of literature suggests that participants might fail to detect delays below $120-200 \mathrm{~ms}$, other studies report that some users are able to spot a latency as small as $50 \mathrm{~ms}$ in the context of mouse-cursor movements [Raaen et al. 2014], and even a latency of around $15 \mathrm{~ms}$ while using a Head-Mounted Display [Mania et al. 2004]. Moreover, when asked to detect changes of system latency while wearing a Head-Mounted Display, users' perceptual stability across different virtual environments would require latencies below $16 \mathrm{~ms}$ [Ellis et al. 2004]

Delays between the execution of an action and its visual feedback can also impair two components of bodily self consciousness, namely the sense of agency (i.e., the sense to have caused the action) and the sense of ownership over one's body (i.e., the sense that my body belongs to me) (e.g., [Gallagher 2000]). The emergence of the sense of agency is though to be based on an internal forward model, which compares the predicted sensory consequences of the motor commands with the actual sensory feedback [Wolpert et al. 1995; Frith et al. 2000]. A mismatch between predicted and observed feedback, as in the case of delayed feedback, can result in a loss of the sense of agency [Longo and Haggard 2009]. When this happens, people might even attribute the observed delayed feedback of their movement to an external cause [Farrer and Frith 2002; Franck et al. 2001]. Although the sense of agency tends to decrease with increasing delay between an action and its sensory 
counterparts [Farrer et al. 2013; Imaizumi and Asai 2015], a certain amount of delay is acceptable for sense of agency to persist. Literature suggests that, when observing a delayed image of one's own hand, people attribute the observed movements to the self even at delays around $150 \mathrm{~ms}$ [Franck et al. 2001; Longo and Haggard 2009; Tsakiris et al. 2006; Tsakiris et al. 2010]. Above 100-150 ms, perceived agency toward the delayed visual feedback of hand movements decreases [Franck et al. 2001], and does not emerge at delays around $500 \mathrm{~ms}$ [Longo and Haggard 2009; Tsakiris et al. 2010]. However, when presented with visual flashes occurring after a voluntary button press, subjects report agency for flashes lagging even up to around $500 \mathrm{~ms}$ [Rohde et al. 2014a]. It has been suggested that body ownership might allow a narrower temporal window of asynchrony than sense of agency, emerging with delays up to 100$150 \mathrm{~ms}$ [Imaizumi and Asai 2015; Longo and Haggard 2009].

A perceivable delay between movements and their observed consequences does not only impair sense of ownership and agency, but may also affect motor performance in 2D and 3D tasks (e.g., [Teather et al. 2009]). For instance, when controlling a character in computer games, delays greater than $150 \mathrm{~ms}$ affect performance [Jörg et al. 2012]. A latency of around $170 \mathrm{~ms}$ led to a longer time necessary to complete a grasping task [Chung and So 1999]. In a simple coordination task, the introduction of a $200 \mathrm{~ms}$ delay significantly increases the error rate [Gutwin 2001]. Similarly, in a tracking task, latencies of or above $110 \mathrm{~ms}$ dramatically increase the error rate [Pavlovych and Stuerzlinger 2011]. A delay around 70$75 \mathrm{~ms}$ has been found already effective in decreasing performance in a VR reaching task [Ware and Balakrishnan 1994], and in tasks which require moving a mouse cursor to a target [MacKenzie and Ware 1993]. In a non-VR and low latency scenario with a Fitts' law style pointing task a latency of about $16 \mathrm{~ms}$ already seemed to have an effect [Friston et al. 2016]. Samaraweera et al. [2013] showed in an experiment using a HMD that inducing a latency of $225 \mathrm{~ms}$ can change gait patterns. In another study they showed that latency as well as using a virtual mirror can alter gait behavior by adding $200 \mathrm{~ms}$ of delay to the system latency to one half of the participants' avatar [Samaraweera et al. 2015].

To summarize, these findings show that the impact of latency differs in our four variables of interest - perceived latency, sense of agency, sense of ownership, and motor performance-for different setups and for different motor tasks.

\section{Methods}

\subsection{Participants}

Ten naïve participants ( 4 males, mean age $\mathrm{M}=23.2$, standard deviation $\mathrm{SD}=2.2$, 9 right handed) with normal or corrected to normal vision took part in the study. Participants provided written informed consent and got paid for their participation. The study was conducted in accordance with the Declaration of Helsinki, and had ethical approval from our University's ethics committee.

\subsection{Apparatus}

We conducted the experiments in a VR setup designed for full-body motor learning of complex actions based on a two sided CAVE (Lshape, $3 \mathrm{~m} \times 2.3 \mathrm{~m}$ for each side). Each wall was operated by two projectors (Projection Design F35 WQ), which ran at $60 \mathrm{~Hz}$. Each of them had a resolution of $2100 \times 1600$ pixels. To separate the images for both eyes, we used passive filters by INFITEC. All four projectors were driven by a self-developed rendering engine running on a single computer to minimize latency. For each wall of the CAVE, we used one NVIDIA Quadro K5000 graphics card.
The rendering engine, which ran at around $240 \mathrm{fps}$, received the position and orientation of the participants' glasses for perspective adaptation as well as joint rotations of 19 joints and information on limb lengths of the participants. We obtained this information by using a passive marker-based full-body motion capture system by OptiTrack. To attach the markers to participants, we used a partly customized tight fitting marker suit consisting of a sleeveless shirt and trousers. We attached markers to this suit or directly to the participants' skin depending on the desired marker position. The motion capture setup consisted of ten Prime $13 \mathrm{~W}$ cameras, which proved to obtain latencies comparable to the commonly installed VICON cameras [Waltemate et al. 2015].

Participants were placed inside a virtual room in front of a virtual mirror. This mirror showed a reflection of the room itself as well as a virtual avatar. This "mirror avatar" was scaled according to the participants' limb lengths and was animated according to the participants' motion in real time. Figure 1 (left) shows an example of this setup.

The end-to-end latency between the participants' movements and the corresponding movements of the mirror avatar was increased synthetically using a FIFO buffer holding back the motion data. This buffer was filled with incoming motion capture frames, and as soon as one of these frames was older than the desired latency offset, it was emitted and used to animate the mirror avatar. If multiple frames satisfied this condition, the one closest to the desired latency was used. We only delayed the mapping of the participants' motion onto the mirror avatar. The movement of the tracked glasses used for perspective adaptation was not delayed.

\subsubsection{Verifying latency}

To determine the latency of our system itself and of our mechanism to induce additional latency, we conducted end-to-end latency measurements. To this end, we used an extension of a well-established latency measurement approach, as described in [Waltemate et al. 2015]: We equipped a test person with motion capture markers and placed this person inside the CAVE. There, the person was instructed to move one arm up and down. This movement was then mapped onto the mirror avatar. We recorded the person together with the rendered mirror avatar using a consumer camera (Nikon 1 J4), which records $400 \mathrm{fps}$. In the resulting video, we measured the delay between the person's movement and the corresponding movement of the mirror avatar by frame counting. To reduce potential errors (e.g., due to manual labeling), we averaged latency measurements over multiple trials. These measurements were performed for the base latency as well as for the latencies that we manually induced during the experiment. We were able to obtain a mean for the base latency of $44.9 \mathrm{~ms}(\mathrm{SD}=6.1)$. The latencies induced manually by our system were identical to the desired latencies.

The base latency of about $45 \mathrm{~ms}$ consists of the individual latencies of the tracking cameras ( $\sim 4 \mathrm{~ms}$ according to manufacturer), of the tracking software, the motion preprocessing $(\sim 2 \mathrm{~ms})$, the network communication $(\sim 1 \mathrm{~ms})$, as well as rendering $(\sim 4 \mathrm{~ms}$ at $240 \mathrm{fps})$, display synchronization, and display hardware $(\sim 19 \mathrm{~ms}$ according to manufacturer). The known latencies add up to $\sim 30 \mathrm{~ms}$, thus the remaining $\sim 15$ ms must be mainly due to display synchronization and tracking software.

\subsection{Procedure and Stimulus}

First, participants filled in questionnaires for demographic data, simulator sickness [Kennedy et al. 1993] and immersive tendencies [Witmer and Singer 1998]. Then they read the instructions for the experiment. Afterwards, they put on the marker suit and per- 


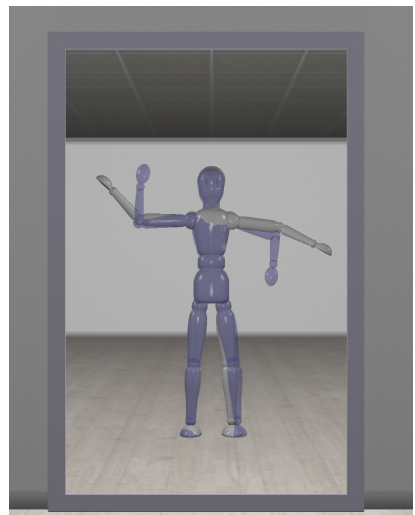

(a)

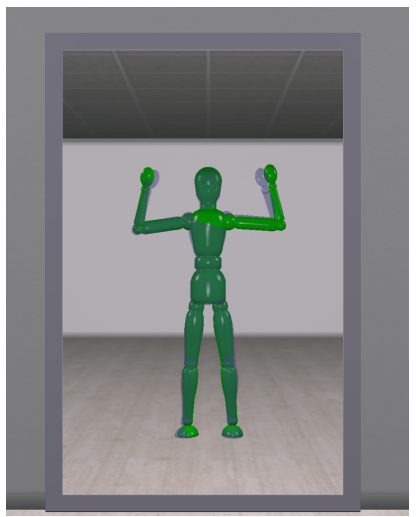

(b)
Figure 2: The participants' avatar is grey (a), while it is not sufficiently close to the target posture of the ghost character (blue character) and green (b), when the posture is fitted sufficiently.

formed skeleton calibration in the CAVE. We did some refinement of marker positions if necessary. Next, participants were presented with the virtual mirror showing the mirror avatar (cf. Figure 1, left). This scene was shown for about one minute to let participants familiarize themselves with the virtual mirror concept.

The following main part of the experiment consisted of multiple trials. In each trial, the mirror avatar was super-imposed with a second character (blue, semi-transparent), which was scaled the same way as the mirror avatar (cf. Figure 2(a)). This "ghost character" performed pre-recorded animations (cf. Figure 3). Participants were instructed to simultaneously mimic the movements of the ghost character, i.e., to move together with the ghost character. For all frames in which the difference in posture between both characters did not exceed a certain threshold (see Section 3.4.2), the mirror avatar was colored green to give feedback on the accuracy of the performance (cf. Figure 2(b)). During the trials, we systematically introduced a delay in the movements of the mirror avatar. This delay was varied between 45 and $350 \mathrm{~ms}$ on a logarithmic scale and randomly set per trial. After each trial the virtual mirror was disabled and turned white, and participants were asked three questions on perceptual judgments (cf. Section 3.4.1). The supplemental video shows an example of one trial. After the main part of the experiment, participants filled in the simulator sickness questionnaire again and took off the marker suit. In total the experiment took about two hours.

We used five different pre-recorded animation sequences to animate the ghost character (cf. Figure 3 and supplemental video). We limited these animations to upper body movements to keep the task simple and avoid fatigue. Pilot experiments had shown that large movements of the whole body including the legs (e.g., squat movement), are too exhausting for a long experiment as ours. Additionally, we noticed that lower body movements that involve lifting one leg off the ground increase the risk of participants tumbling. The same movement was always presented twice in a row per trial. The movements varied in speed and trajectory and thus were not entirely predictable. This kind of movement was selected in order to prevent participants from recurring to unwanted strategies while performing the motor task. Indeed, individuals tend to modify their performance in order to compensate for feedback delays either by slowing down or by adopting a "move and wait" strategy [Ferrell 1965; Park and Kenyon 1999]. The latter consists of ignoring the visual feedback while executing fast movements, and then waiting to catch up with the visual feedback before continuing the task. By forcing participants to execute fast movements with variable velocity and non-linear paths, we aimed to prevent such strategies [Rohde et al. 2014b; Rohde and Ernst 2016]. Each animation lasted $5 \mathrm{~s}$ and had the same starting posture (cf. Figure 3(a)). Before an animation started, the ghost character remained in the starting posture for $1.5 \mathrm{~s}$ to allow participants to take that posture. Thus, each trial lasted $6.5 \mathrm{~s}$. As latency offsets we used $0,30,80,165$ and $305 \mathrm{~ms}$. When added to the baseline latency of the system, these offsets resulted in end-to-end latencies of 45, 75, 125, 210 and $350 \mathrm{~ms}$, respectively. We decided to take also large latencies $(>125 \mathrm{~ms})$ into account to identify the perceptual limits of the sense of agency and body ownership, as pilot experiments had revealed that participants tolerate larger delays when judging agency and ownership.

The experiment included 200 trials and ten practice trials. In the practice trials each animation sequence was shown two consecutive times with no additional latency. In the other 200 trials we randomized animations as well as latency offsets, while still making sure to equally distribute the combinations of animation sequences and latency offsets, so that each combination occurred equally often. Therefore, each animation as well as each latency was presented 40 times. Since this results in a long time for participants to stand and perform the movements (mean duration $=68 \mathrm{~min}(\mathrm{SD}=20)$ ), we included a short break every 20 trials. These breaks were optional and participants could continue with the experiment whenever they wanted by touching a virtual box. Additionally, the mean accuracy of the motor performance since the last break was shown to motivate participants.

\subsection{Dependent Variables}

\subsubsection{Perceptual Judgments}

After each of the 200 trials participants were asked the following three questions in randomized order:

Agency judgment (AJ): Was the motion of the grey avatar the visual feedback to the movement you just performed?

Simultaneity judgment (SJ): Was the motion of the grey avatar simultaneous to your movement?

Ownership judgment (OJ): Did you feel that the grey avatar belonged to you?

For agency judgment we instructed participants that the motion performed by their avatar could either reflect their own movement or some pre-recorded movement previously performed by them or some previous participant. We introduced such a "cover story" to provide participants with a reasonable alternative when attributing the causality of the action [Rohde and Ernst 2016]. To avoid that participants probe the system for agency (e.g., by making unexpected movements to test if the avatar follows), we instructed them explicitly against such probing. We asked participants to answer the questions according to how they felt and how they perceived the movements of their avatar. The questions were visualized as text inside the CAVE and were answered by touching virtual "Yes" or "No" boxes. The fact that AJ, OJ and SJ were all yes/no tasks allowed a direct comparison of the temporal windows of perceiving agency, ownership, and simultaneity [Rohde et al. 2014a].

\subsubsection{Motor Performance}

The motor performance was determined by checking the posture deviation of the participants' avatar with the ghost character. For each frame the distances of shoulder, elbow, and wrist joint of the mirror avatar to the corresponding joints of the ghost character were 


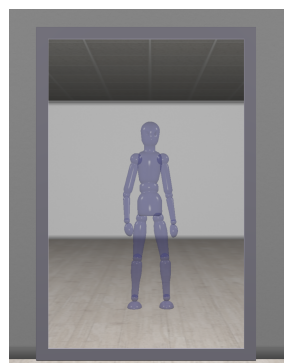

(a)

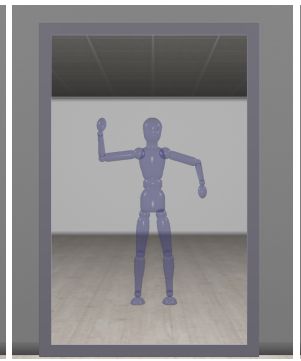

(b)

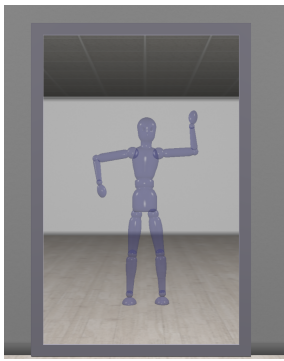

(c)

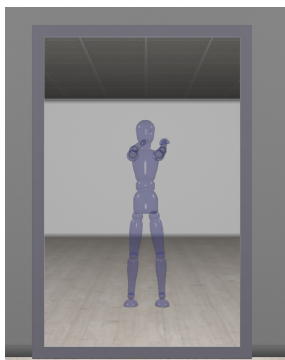

(d)

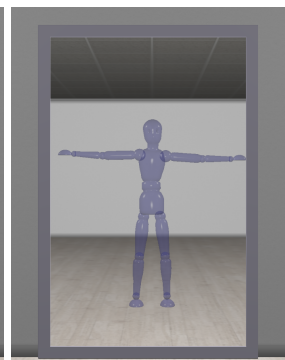

(e)

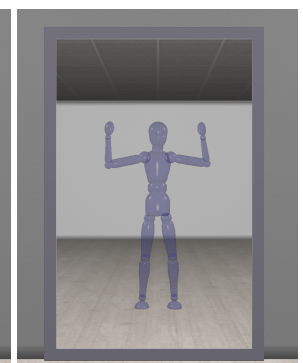

(f)

Figure 3: (a): Starting posture. (b)-(f): Turning point postures of the five animations used for the ghost character.

calculated and averaged. If that average distance was inside the error range of $12 \mathrm{~cm}$, the participants' posture was counted as sufficiently close and successful. The number of successful frames divided by all rendered frames gave us the percentage of successful postures in each trial.

\section{Results}

The first ten trials served as practice trials and were not included in the analyses. For each participant we calculated the percentage of "yes" responses in each perceptual task (i.e., AJ, OJ, SJ). The effect of feedback delay on perceptual judgments and motor performance was assessed separately for each task, by fitting the probability of "yes" responses and the accuracy in the motor performance with a generalized linear mixed model (GLMM, [Agresti 2002; Moscatelli et al. 2012]). In such a model, the overall variance is divided into a fixed and a random component [Agresti 2002]. The fixed component tests the effect of the independent variable (manipulated in the experiment), while the random effect accounts for the heterogeneity among different participants. Thus, the GLMM allows the analysis of clustered categorical data, at the population and individual levels simultaneously [Moscatelli et al. 2012]. Here, feedback delay (log-transformed) was the fixed effect, and subject identity was the random effect (Model 1). A probit link function was applied. The model parameters were estimated using Maximum Likelihood Estimation (MLE). The data was analyzed with MATLAB. The GLMM analysis revealed a significant effect of feedback delay for all tasks (all $\mathrm{p}<0.001$ ).

As shown in Figure 4, the higher the delay, the lower the probability of responding "yes" in the perceptual judgment tasks, and the lower the accuracy in motor performance (see also Table 1 for results for each tested delay). In other words, sense of agency, ownership, and perceived simultaneity decreased, and motor performance worsened, with increasing delay. Regarding the perceptual judgment tasks (Figure 4(a)), the temporal window of perceived simultaneity was narrower than those of perceived agency and ownership. Trials with the minimum system delay ( $45 \mathrm{~ms}$ ) were perceived as simultaneous $88 \%$ of the times on average across participants. Perceived simultaneity dramatically dropped with increasing delay: $49 \%$ of trials with $210 \mathrm{~ms}$ delay and only $12 \%$ of trials presenting $350 \mathrm{~ms}$ delay were perceived as simultaneous.

In contrast, although both perceived agency and ownership decreased significantly with increasing delay, they did not break down even at the highest tested delay $(350 \mathrm{~ms})$. Indeed, while for minimum delay participants reported sense of agency in $96 \%$ of the trials and sense of ownership in $97 \%$ of them, sense of agency and ownership still occurred at $350 \mathrm{~ms}$ delay in $71.5 \%$ and $62 \%$ of the trials, respectively. Responses in the $\mathrm{OJ}$ and AJ tasks were com- parable, as shown by overlapping confidence intervals (CI, set at $95 \%$ confidence level) for all tested delays between the two tasks (see Figure 4(a) and Table 1). Motor performance gradually worsened with increasing delay, from $66 \%$ of accuracy at $45 \mathrm{~ms}$ delay to $36 \%$ at $350 \mathrm{~ms}$ (Figure 4(b) and Table 1). Overall, performance in each of the four tasks did not differ between 45 and $75 \mathrm{~ms}$ delays, as shown by overlapping $\mathrm{CI}$ in each task between those two delays. Motor performance significantly worsened between $75 \mathrm{~ms}(\mathrm{M}=64.8 \%, \mathrm{CI}=58-71.6)$, and $125 \mathrm{~ms}(\mathrm{M}=61.4 \%$, $\mathrm{CI}=54.3-68.7$; Wilcoxon signed-rank test, $\mathrm{p}=0.009)$. Similarly, perceived simultaneity showed a tendency to decrease between $75 \mathrm{~ms}(\mathrm{M}=87 \%, \mathrm{CI}=83-90)$ and $125 \mathrm{~ms}(80 \%, \mathrm{CI}=76-$ 84 , Wilcoxon signed-rank test, $\mathrm{p}=0.07$ ). Conversely, perceived agency and ownership started declining between 125 and $210 \mathrm{~ms}$, while they did not significantly differ between $75 \mathrm{~ms}$ and $125 \mathrm{~ms}$ (agency, $75 \mathrm{~ms}: \mathrm{M}=94.8 \%, \mathrm{CI}=92-96.7 ; 125 \mathrm{~ms}: \mathrm{M}=94 \%$, $\mathrm{CI}=91.2-96.1$, Wilcoxon signed-rank test, $\mathrm{p}=1$; ownership, $75 \mathrm{~ms}: \mathrm{M}=95.5 \%, \mathrm{CI}=93-97.3 ; 125 \mathrm{~ms}: \mathrm{M}=96.5 \%, \mathrm{CI}=94-$ 98 , Wilcoxon signed-rank test, $\mathrm{p}=0.52$ ).

With the minimum delay (i.e., $45 \mathrm{~ms}$ ), motor performance was more accurate in trials reported as simultaneous (accuracy, $\mathrm{M}=67.3 \%, \mathrm{SD}=11.9)$ than in those perceived as nonsimultaneous $(\mathrm{M}=54.2 \%, \mathrm{SD}=10.3)$, and in those for which participants perceived agency $(\mathrm{M}=66.5 \% \mathrm{SD}=12.3)$ and ownership $(M=66.5 \%, S D=23)$ as compared to those that did not elicit agency $(\mathrm{M}=62 \%, \mathrm{SD}=10.7)$ and ownership $(\mathrm{M}=51.7 \%$, $\mathrm{SD}=10.5$ ) feelings.

Given the positive association between the quality of the motor performance and the successive perceptual judgements, we tested a second model to assess whether the influence of motor performance in the task was significant. We fitted the probability of "yes" responses in each task with a GLMM model with performance accuracy as fixed effect, and subject identity as random effect (Model 2 ). Results showed that motor performance accuracy predicts responses in all tasks (all $\mathrm{p}<0.001$ ). We compared the results of Model 2 (i.e., the one with performance accuracy as a fixed effect) with those of Model 1 (i.e., the one with feedback delay as a fixed effect). The two models employ the same functions, and differ in the predictors only. To select the model that better fits the data (i.e., the fixed effects that better explain the results), we compared the two models using the Akaike Information Criterion (AIC, [Akaike 1973]). According to the AIC, Model 2 fitted the data better than Model 1. This result suggests that, although both delay and motor performance accuracy affect perceptual judgments, participants rely more on their own performance than on the delay itself when estimating feedback simultaneity, sense of agency, and ownership. However, we cannot exclude that the relationship between performance accuracy and subjective judgments might be mediated by a 


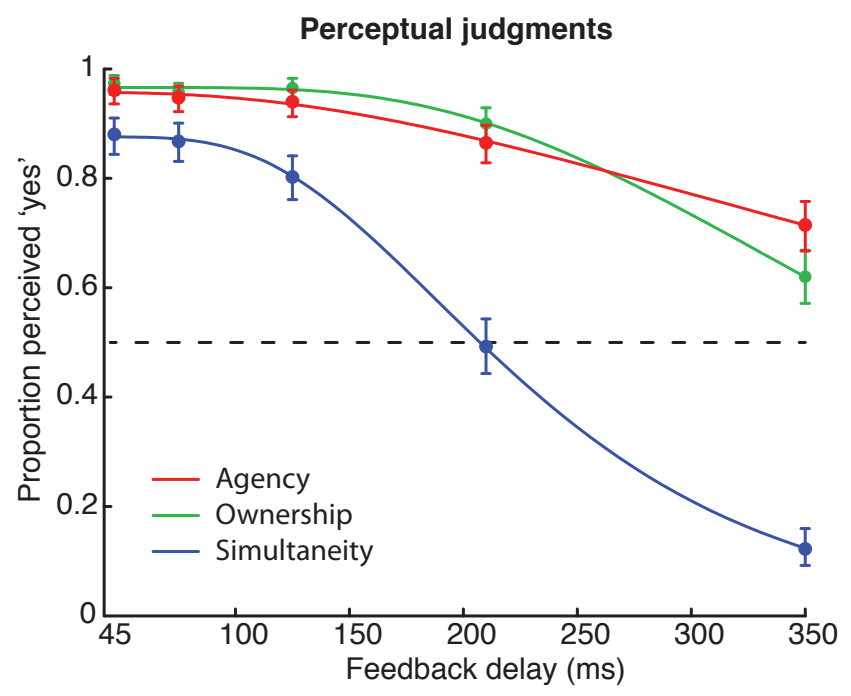

(a)

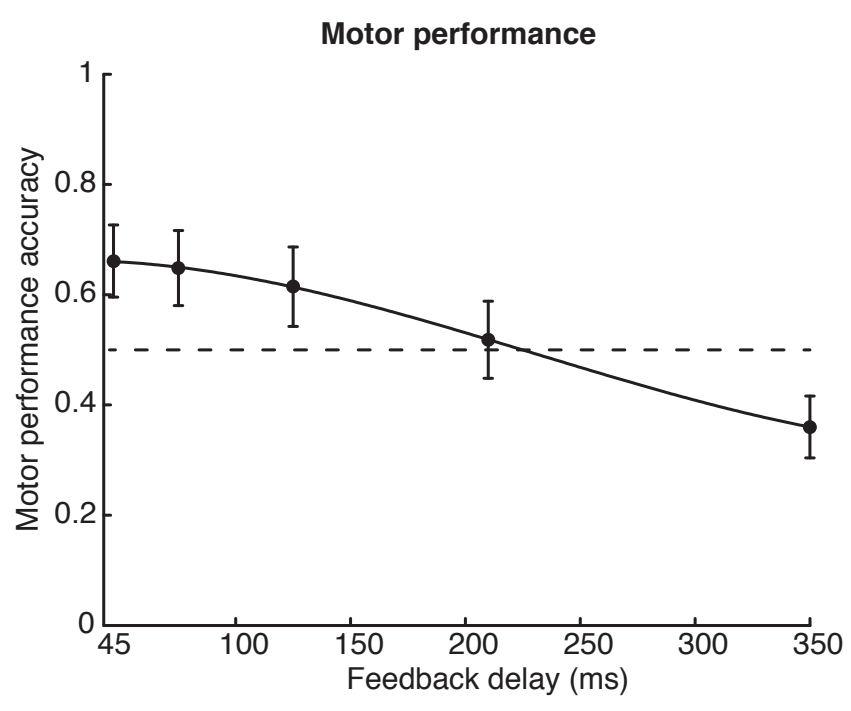

(b)

Figure 4: Psychometric functions for all tasks, obtained by pulling together the data from all participants. a) Perceptual judgments (agency, ownership, simultaneity). b) Motor performance. Analyses were performed on the individual data from each participant, while aggregated data are shown for illustrative purposes only. Error bars represent confidence intervals set at $95 \%$.

third latent variable, and further studies are necessary to better understand this issue.

To make sure that participants were not influenced or biased because they were asked the same questions repeatedly in the $\mathrm{AJ} / \mathrm{SJ} / \mathrm{OJ}$ tasks, we ran further analyses. For each perceptual task we fitted the probability of "yes" responses with a GLMM with feedback delay, trial number, and their interaction as fixed effects, and subject identity as random effect (Model 3). The only significant fixed effect was that of the factor feedback delay (all $\mathrm{p}<$ 0.001 ), while trial number and the interaction of delay and trial number were not significant (all $\mathrm{p}>0.07$ ). Moreover, a model comparison according to the AIC favoured a model with a fixed effect only for the delay (Model 1) over more complex models with also fixed effects of trial number or the interaction between trial number and feedback delay (Model 3). These findings show that participants did not alter their response strategy during the time of the experimental session.

To assess whether individual differences in immersive tendencies are related to individual differences in perceptual and motor tasks, we calculated Pearson correlation between each item of the immersive tendencies questionnaire (ITQ) and the performance in each task with minimum delay (i.e., $45 \mathrm{~ms}$ ). We found a significant correlation between the item of the ITQ ["Do you easily become deeply involved in movies or TV dramas?"] and sense of ownership $(r=0.66, p=0.038)$, and between the same item and sense of agency $(r=0.81, p=0.005)$. Thus, participants who report to be usually deeply involved in movies are the ones showing greater sense of agency and ownership in the experimental task. Moreover the item ["Do you ever become so involved in doing something that you lose all track of time?"] was negatively correlated with perceived simultaneity $(\mathrm{r}=-0.73, \mathrm{p}=0.01)$ and motor performance $(\mathrm{r}=-0.65, \mathrm{p}=0.04)$. Thus, participants reporting a tendency to lose track of time showed worse motor performance and a higher tendency to report trials at $45 \mathrm{~ms}$ delay as non-simultaneous.

Finally, to test for the presence of motion sickness induced by our system, we compared the responses to each item of the simulator sickness questionnaire between the first and the second presentation of the questionnaire using the Wilcoxon Signed rank test. Participants did not show motion sickness after taking part in the experiment (all $\mathrm{p}>0.38)$.

\section{Discussion}

Previous research involving hand movement suggests that sense of ownership might be more affected by delay than sense of agency [Imaizumi and Asai 2015]. Here we found that even a delay as high as $350 \mathrm{~ms}$ elicits both sense of agency and ownership. This result is in line with recent findings suggesting that sense of agency toward a moving hand might drive sense of ownership even toward the delayed image of the hand [Asai 2016]. Our findings suggest that movements which are more complex than a simple button press, as in the case of the full-body movements described in our study, induce a strong sense of ownership, even despite long delays in the visual feedback. Notably the temporal structure of such a feedback would be strongly correlated with participants' motor performance. Thus, participants might rely on correlation between the temporal structure of the motor and visual signals to infer a common cause (between motor actions and visual feedback), and a sense of agency, ownership, and simultaneity. A recent computational model for multisensory integration [Parise and Ernst 2016] suggests that people rely on such temporal correlation across different sensory signals when estimating simultaneity and common source.

Furthermore, results showed that motor performance accuracy predicts perceptual judgments even better than delays itself. Thus, when motor performance is poor (i.e., in case of a mismatch between the desired outcome and the actual performance), participants tend to perceive the feedback as non-simultaneous, and to lose sense of agency and ownership. In other words, a small delay tends to be perceived as non-simultaneous in case of high performance errors, and higher delays might go undetected if motor performance is accurate. This finding suggests that, in principle, the same delay in a VR system might or might not impair perceptual judgments, depending on the difficulty of the motor task. In case of a simple motor task, leading to high performance accuracy, the delay might 


\begin{tabular}{ccccc}
\hline Delay & Agency & Ownership & Simultaneity & Motor performance \\
\hline \multirow{2}{*}{$45 \mathrm{~ms}$} & $\begin{array}{c}96 \% \\
(93.6-97.7 \%)\end{array}$ & $\begin{array}{c}97.25 \% \\
(95.5-98.8 \%)\end{array}$ & $\begin{array}{c}88 \% \\
(84.4-91 \%)\end{array}$ & $\begin{array}{c}66.1 \% \\
(59.6-72.6 \%)\end{array}$ \\
\hline $75 \mathrm{~ms}$ & $94.8 \%$ & $95.5 \%$ & $86.75 \%$ & $64.83 \%$ \\
& $(92-96.7 \%)$ & $(93-97.3 \%)$ & $(83-90 \%)$ & $(58-71.6 \%)$ \\
\hline \multirow{2}{*}{$125 \mathrm{~ms}$} & $94 \%$ & $96.5 \%$ & $80.25 \%$ & $61.5 \%$ \\
& $(91.2-96.1 \%)$ & $(94-98 \%)$ & $(76-84 \%)$ & $(54.3-68.7 \%)$ \\
\hline $210 \mathrm{~ms}$ & $86.5 \%$ & $90 \%$ & $49.25 \%$ & $51.83 \%$ \\
& $(82.8-89.7 \%)$ & $(86.6-92.8 \%)$ & $(44.35-54.3 \%)$ & $(44.7-58.9 \%)$ \\
\hline $350 \mathrm{~ms}$ & $71.5 \%$ & $62 \%$ & $12.25 \%$ & $36 \%$ \\
& $(66.8-75.9 \%)$ & $(57-66.8 \%)$ & $(9.2-15.9 \%)$ & $(30.4-41.6 \%)$ \\
\hline
\end{tabular}

Table 1: The table depicts the mean percentage of "yes" answers for agency, ownership, and simultaneity judgments as well as mean percentage of the successful postures for motor performance for each delay. The confidence intervals (lower limit-upper limit), set at $95 \%$, are listed beneath their corresponding mean value.

go unnoticed. In case of more complex tasks, leading to higher chances of performance errors, delays will exert a stronger impact on perceived agency, ownership, and simultaneity. This might explain why in previous studies recurring to button press [Rohde et al. 2014a], participants were unaware even of long delays: a simple motor performance requiring only a button press would not lead to error signals related to the motor performance, and this in turn reduces the probability of detecting the delay. For the same reason, trained users (for instance, experts in a certain sport), performing a motor task better than novices in a sport scenario, might be perceptually less affected by delays than novices. Previous studies have shown that people are not particularly accurate in detecting delays in sensorimotor tasks: delays up to around $200-250 \mathrm{~ms}$ can even go unnoticed, and in some cases participants might report agency also for events preceding the action [Rohde et al. 2014a]. Here we show that the quality of the motor performance strongly influences perceptual decisions.

\section{Conclusion}

In our study, we used a novel paradigm employing VR and psychophysical procedures to investigate how delays between an action and its visual feedback affect perception and motor performance. In particular, we assessed the impact of latency on perceived simultaneity, sense of agency, ownership, as well as motor performance during the execution of full-body movements in front of a virtual mirror. Considering the large variability in the literature concerning a possible threshold for visual delays to be detected and affect behavior, we systematically assessed the effect of delays on motor performance and perceptual judgments in the same setup, and in the same experimental session. To this end, we parametrically varied the delay of the visual feedback between $45 \mathrm{~ms}$ (i.e., the latency intrinsic in the system) and $350 \mathrm{~ms}$, while participants on each trial performed both a motor task and perceptual judgments on the delayed feedback. Results showed that, although inducing delay increasingly affects perceptual judgments and motor performance, incrementing the delay from 45 to $75 \mathrm{~ms}$ had no significant impact on participants' performance in the four tasks. Delays above $75 \mathrm{~ms}$ worsen motor performance and influence simultaneity perception, while they do not affect perceived agency and ownership, which significantly start declining only later, between $125 \mathrm{~ms}$ and $210 \mathrm{~ms}$. Interestingly, the temporal windows of perceived agency and ownership are much broader than the one of perceived simultaneity: while at the highest tested delay (i.e., $350 \mathrm{~ms}$ ) visual feedback is hardly perceived as simultaneous, perceived agency and ownership are not disrupted, although significantly reduced. Thus, participants tolerated perceptible delays when reporting agency and ownership toward their avatar's movements. These findings are in line with studies investigating the temporal window of agency and simultaneity [Farrer et al. 2013; Rohde et al. 2014a] when participants judged whether a visual event presented on a screen was caused by their button press and whether it was simultaneous. Here we show that this also holds true in the case of complex upper-body movements. Moreover, we found that individual differences in immersive tendencies modulate sensitivity to delays, sense of agency, and ownership, as well as motor performance.

In conclusion, our study extends our understanding of the effect of feedback delays on perceptual and motor tasks. While previous studies mainly involved manual tasks, we focused on more complex movements involving the entire upper body. Since we always tracked and visualized the whole body in an immersive environment employing a virtual mirror, we assume that our findings transfer from upper-body to full-body movements, but this has to be examined in future studies. Moreover, our study introduced a systematic investigation of the interplay between perceived simultaneity, agency, and ownership in virtual environments, while previous research has mainly focused on a subset of these phenomena. The present findings contribute to a deeper understanding of what information people rely on when making perceptual decisions about agency, ownership, and simultaneity. Furthermore, it contributes to fill a gap in the literature regarding which delay might be considered acceptable for VR systems for motor learning of complex tasks involving full-body movements. While smaller delays might not appreciably affect perception, they might still impair motor performance.

In general, this work stresses the importance of latency for fullbody interaction in virtual environments. While latency is often neglected or at least put in second place, we argue that the latency of a VR system used for a specific study should at least be reported. Moreover, the effect of latency should be taken into consideration as the source of possible side effects.

\section{Future Work}

In future work we would like to examine the influence of jitter in the latency on motor performance, simultaneity perception, sense of ownership, and sense of agency. As we concentrated on upperbody movements in the present study we would like to investigate whether the findings of this work also hold true for interaction involving complex movements of the full body. Moreover, we would like to investigate the impact of appearance on sense of embodi- 
ment in the virtual mirror scenario. To this end, we will employ 3D-scanning to create high-quality avatars of participants, so that we can confront them with a more realistic virtual mirror image.

\section{Acknowledgements}

This research was supported by the Cluster of Excellence Cognitive Interaction Technology CITEC (EXC 277) at Bielefeld University, which is funded by the German Research Foundation (DFG).

\section{References}

Agresti, A. 2002. Categorical Data Analysis. Wiley Series in Probability and Statistics. Wiley.

AKAIKE, H. 1973. Information theory and an extension of the maximum likelihood principle. In Proc. of Second International Symposium on Information Theory, 267-281.

AsAI, T. 2016. Agency elicits body-ownership: proprioceptive drift toward a synchronously acting external proxy. Experimental brain research 234, 5, 1163-1174.

ChUnG, K., AND So, R. H. 1999. Effects of hand movement lag on discrete manual control tasks in virtual environements. In Proceedings of the Human Factors and Ergonomics Society Annual Meeting, vol. 43, SAGE Publications, 1210-1213.

De KoK, I., Hough, J., Hülsmann, F., Botsch, M., SCHLANGEN, D., AND KopP, S. 2015. A multimodal system for real-time action instruction in motor skill learning. In Proc. of International Conference on Multimodal Interaction, ACM, $355-362$.

Ellis, S. R., Mania, K., Adelstein, B. D., And Hill, M. I. 2004. Generalizeability of latency detection in a variety of virtual environments. In Proceedings of the Human Factors and Ergonomics Society Annual Meeting, vol. 48, SAGE Publications, 2632-2636.

FARRER, C., AND FRITH, C. 2002. Experiencing oneself vs another person as being the cause of an action: The neural correlates of the experience of agency. NeuroImage 15, 3, 596-603.

Farrer, C., Valentin, G., And Hup, J. 2013. The time windows of the sense of agency. Consciousness and Cognition 22, $4,1431-1441$.

FERRELl, W. R. 1965. Remote manipulation with transmission delay. IEEE Transactions on Human Factors in Electronics, 1, 24-32.

Franck, N., Farrer, C., GeorgiefF, N., Marie-Cardine, M., DAlry, J., D’Amato, T., AND JeAnnerod, M. 2001. Defective recognition of one's own actions in patients with schizophrenia. American Journal of Psychiatry 158, 3, 454-459.

Friston, S., Karlström, P., And Steed, A. 2016. The effects of low latency on pointing and steering tasks. IEEE transactions on visualization and computer graphics $22,5,1605-1615$.

Frith, C. D., Blakemore, And Wolpert, D. M. 2000. Abnormalities in the awareness and control of action. Philosophical Transactions of the Royal Society of London B: Biological Sciences 355, 1404, 1771-1788.

Gallagher, S. 2000. Philosophical conceptions of the self: implications for cognitive science. Trends in Cognitive Sciences 4, $1,14-21$.
Gonzalez-Franco, M., Perez-Marcos, D., Spanlang, B., AND SLATER, M. 2010. The contribution of real-time mirror reflections of motor actions on virtual body ownership in an immersive virtual environment. In Proceedings of IEEE Virtual Reality, 111-114.

Gutwin, C. 2001. The effects of network delays on group work in real-time groupware. In Proc. Seventh European Conference on Computer-Supported Cooperative Work, Springer, 299-318.

Held, R., AND Durlach, N. 1991. Telepresence, time delay and adaptation. In Pictorial communication in virtual and real environments. 232-246.

ImAIZUMI, S., AND ASAI, T. 2015. Dissociation of agency and body ownership following visuomotor temporal recalibration. Frontiers in Integrative Neuroscience 9, 35.

Jörg, S., Normoyle, A., And Safonova, A. 2012. How responsiveness affects players' perception in digital games. In Proceedings of the ACM Symposium on Applied Perception, 3338.

Kawabe, T., Roseboom, W., And Nishida, S. 2013. The sense of agency is action-effect causality perception based on crossmodal grouping. Proceedings of the Royal Society of London B: Biological Sciences 280, 1763.

Kennedy, R. S., Lane, N. E., Berbaum, K. S., And LilienTHAL, M. G. 1993. Simulator sickness questionnaire: An enhanced method for quantifying simulator sickness. The international journal of aviation psychology 3, 3, 203-220.

LONGO, M. R., AND HaGGARD, P. 2009. Sense of agency primes manual motor responses. Perception 38, 1, 69-78.

Lugrin, J.-L., LANDeck, M., And Latoschik, M. E. 2015. Avatar embodiment realism and virtual fitness training. In Proceedings of IEEE Virtual Reality, 225-226.

MacKenzie, I. S., And Ware, C. 1993. Lag as a determinant of human performance in interactive systems. In Proceedings of the INTERACT'93 and CHI'93 conference on Human factors in computing systems, ACM, 488-493.

Mania, K., Adelstein, B. D., Ellis, S. R., And Hill, M. I. 2004. Perceptual sensitivity to head tracking latency in virtual environments with varying degrees of scene complexity. In Proc. of the 1st Symposium on Applied Perception in Graphics and Visualization, ACM, 39-47.

Mauve, M., Vogel, J., Hilt, V., And Effelsberg, W. 2004. Local-lag and timewarp: providing consistency for replicated continuous applications. IEEE Transactions on Multimedia 6, $1,47-57$.

Meehan, M., Razzaque, S., Whitton, M. C., And Brooks, F. P. 2003. Effect of latency on presence in stressful virtual environments. In Proceedings of IEEE Virtual Reality, 141-148.

Moscatelli, A., Mezzetti, M., And Lacquaniti, F. 2012. Modeling psychophysical data at the population-level: the generalized linear mixed model. Journal of Vision 12, 11, 26-26.

Parise, C. V., AND ERnst, M. O. 2016. Correlation detection as a general mechanism for multisensory integration. Nature communications 7 .

PARK, K. S., AND KENYON, R. V. 1999. Effects of network characteristics on human performance in a collaborative virtual environment. In Proceedings of IEEE Virtual Reality, 104-111. 
Pavlovych, A., And Stuerzlinger, W. 2011. Target following performance in the presence of latency, jitter, and signal dropouts. In Proceedings of Graphics Interface, 33-40.

RAAEN, K., EG, R., AND GRIwodZ, C. 2014. Can gamers detect cloud delay? In Proceedings of the 13th Annual Workshop on Network and Systems Support for Games, IEEE Press.

RohdE, M., AND ERnst, M. O. 2016. Time, agency, and sensory feedback delays during action. Current Opinion in Behavioral Sciences 8, 193-199.

Rohde, M., Scheller, M., And ERnst, M. O. 2014. Effects can precede their cause in the sense of agency. Neuropsychologia 65, 191-196.

Rohde, M., van Dam, L. C., And ERnst, M. O. 2014. Predictability is necessary for closed-loop visual feedback delay adaptation. Journal of Vision 14, 3, 1-23.

Samaraweera, G., Guo, R., AND Quarles, J. 2013. Latency and avatars in virtual environments and the effects on gait for persons with mobility impairments. In IEEE Symposium on $3 D$ User Interfaces, IEEE, 23-30.

Samaraweera, G., Perdomo, A., And Quarles, J. 2015. Applying latency to half of a self-avatar's body to change real walking patterns. In Proceedings of IEEE Virtual Reality, 8996.

Stauffert, J. P., Niebling, F., And Latoschik, M. E. 2016. Reducing application-stage latencies of interprocess communication techniques for real-time interactive systems. In Proceedings of IEEE Virtual Reality, 287-288.

Teather, R. J., Pavlovych, A., Stuerzlinger, W., And MACKenZIE, I. S. 2009. Effects of tracking technology, latency, and spatial jitter on object movement. In Proceedings of IEEE Symposium on 3D User Interfaces, 43-50.

Tsakiris, M., Prabhu, G., And Haggard, P. 2006. Having a body versus moving your body: How agency structures bodyownership. Consciousness and cognition 15, 2, 423-432.

TsAKIRIS, M., LONGo, M. R., AND HagGARD, P. 2010. Having a body versus moving your body: neural signatures of agency and body-ownership. Neuropsychologia 48, 9, 2740-2749.

Waltemate, T., Hülsmann, F., Pfeiffer, T., KopP, S., And BotsCH, M. 2015. Realizing a low-latency virtual reality environment for motor learning. In Proc. of ACM Symposium on Virtual Reality Software and Technology, 139-147.

WARE, C., AND BALAKRishnan, R. 1994. Reaching for objects in VR displays: lag and frame rate. ACM Transactions on Computer-Human Interaction 1, 4, 331-356.

Witmer, B. G., AND Singer, M. J. 1998. Measuring presence in virtual environments: A presence questionnaire. Presence: Teleoperators and virtual environments 7, 3, 225-240.

Wolpert, D. M., GHAhramani, Z., AND Jordan, M. I. 1995. An internal model for sensorimotor integration. Science 269, 5232, 1880-1882.

YArrow, K., SVerdrup-Stueland, I., Roseboom, W., AND ARNOLD, D. H. 2013. Sensorimotor temporal recalibration within and across limbs. Journal of Experimental Psychology: Human Perception and Performance 39, 6, 1678-1689. 